\title{
Enseigner la géographie en territoire protestant allemand : le cas de l'université de Marbourg (1527-1637)
}

\section{Axelle Chassagnette}

\section{OpenEdition}

\section{Journals}

Édition électronique

URL : https://journals.openedition.org/geohist/416

DOI : 10.4000/geohist.416

ISSN : 2264-2617

\section{Éditeur}

Association française de la Revue de géographie historique

\section{Référence électronique}

Axelle Chassagnette, «Enseigner la géographie en territoire protestant allemand : le cas de l'université de Marbourg (1527-1637) », Revue de géographie historique [En ligne], 17-18 | 2020, mis en ligne le 03 novembre 2020, consulté le 12 juin 2021. URL : http://journals.openedition.org/geohist/416 ; DOI : https://doi.org/10.4000/geohist.416

Ce document a été généré automatiquement le 12 juin 2021



$\mathrm{Ce}(\mathrm{tte})$ œuvre est mise à disposition selon les termes de la Licence Creative Commons Attribution Pas d'Utilisation Commerciale - Pas de Modification 4.0 International. 


\title{
Enseigner la géographie en territoire protestant allemand : le cas de l'université de Marbourg (1527-1637)
}

\author{
Axelle Chassagnette
}

Comment transmettre la connaissance que les Européens avaient du monde au début de l'époque moderne? La compréhension et la représentation de la Terre sont profondément modifiées à la Renaissance: dès la seconde moitié du XV siècle, les voyages vers les côtes de l'Afrique occidentale, à travers l'Océan Indien, vers l'Inde puis l'Extrême-Orient, vers l'Amérique d'autre part, transforment une image savante de l'œkoumène - la Terre habitée - jusque là fondée essentiellement sur les textes de l'Antiquité et sur les récits des marchands et des pèlerins médiévaux. Au début du XV ${ }^{\mathrm{e}}$ siècle, la redécouverte du manuscrit grec de la Géographie de Ptolémée (dont le souvenir, mais non le contenu précis, avait été conservé dans l'Occident médiéval), sa traduction puis sa réception élargie sous forme manuscrite puis imprimée, offrent aux savants des techniques de représentation cartographique, un vocabulaire conceptuel ainsi qu'une liste de toponymes à laquelle sont associées des coordonnées géographiques (Gautier-Dalché, 2009). Un domaine de savoir nommé "géographie » apparait sous différentes formes textuelles et graphiques, et se construit autour de références livresques anciennes et nouvelles, ainsi que d'un réservoir croissant d'informations tirées de récits de voyages (Broc, 1986 ; Lestringant, 1991 ; Besse, 2003). La connaissance de la nature et du monde, dont la présentation, au Moyen Âge, s'articulait majoritairement autour de la physique aristotélicienne, de l'astronomie, de l'inventaire naturel et du récit de la Création, peut, à la Renaissance, être structurée par un principe d'organisation directement spatiale, tel que l'adoptent les grandes cosmographies qui sont rédigées et imprimées en nombre au XVI siècle. La géographie tend dès lors à se constituer en discipline : elle dispose de concepts et d'un vocabulaire propres, d'autorités reconnues, de formes d'exposition communes, fait l'objet de manuels, d'ouvrages de synthèse, et de tentatives de définition, notamment dans les 
livres qui portent sur la nature, la caractérisation et l'organisation des savoirs philosophiques. Un autre indice de cette évolution en discipline du savoir géographique est la place croissante qui lui est accordée dans l'enseignement de la Renaissance. Celuici, qui fait l'objet de nombreuses études, s'est rarement arrêté à la géographie, qui n'entre pas dans le canon des disciplines philosophiques du trivium et du quadrivium (pourtant largement bousculé à cette époque), et surtout n'est que rarement évoqué dans les textes normatifs qui fixent l'organisation de l'enseignement dans les universités et les collèges de philosophie européens (Paulsen, 1885; Rüegg, 1996; Hammerstein et Buck, 1996). La chronologie de l'essor de la discipline géographique, d'autre part, échappe en grande partie à celle du paradigme historiographique de la "révolution scientifique », qui se penche plus volontiers sur les transformations que connaissent l'astronomie, la physique et la médecine entre le milieu du XVI ${ }^{\mathrm{e}}$ siècle et la fin du XVII siècle (Shapin, 1998). Néanmoins, les quelques travaux qui traitent de ce sujet montrent que le développement des cours de géographie au XVI ${ }^{\mathrm{e}}$ siècle est réel, et répond souvent à des besoins nouveaux, notamment ceux de la formation des futurs missionnaires, des marchands et des navigateurs (Dainville, 1940; Cormack, 1997; Chassagnette, 2018). L'étude de l'enseignement permet ainsi à l'historien des sciences et des disciplines d'échapper à une reconstitution de l'histoire d'un savoir qui ne s'appuierait que sur les sources philosophiques et normatives, ou encore sur les grandes œuvres et les grands noms, dont l'inventaire permet certes de marquer les jalons de l'invention conceptuelle propre à ce savoir, mais ne donne pas toujours à voir ce que l'ensemble de la communauté des lettrés - quelle que soit leur discipline ou profession - en connaissait, ni l'usage réel qu'elle en avait. Elle permet aussi de comprendre où, et de quelle manière se construit la maitrise de cette nouvelle discipline.

2 Le Saint Empire, bien qu'il n'ait guère participé aux "Grandes découvertes ", est un producteur actif du nouveau savoir géographique dès le $\mathrm{XV}^{\mathrm{e}}$ siècle. Cela s'explique notamment par l'intérêt précoce des humanistes allemands pour les savoirs mathématiques et par leur désir de produire un portrait nouveau de l'Allemagne, qui s'éloigne du discours admiratif, mais condescendant et dépassé, des anciens auteurs latins, souvent repris avec ironie par les humanistes italiens (Gallois, 1890 ; Strauss, 1959). Le projet de Germania illustrata de Conrad Celtis, à la fin du XV siècle, propose ainsi de produire des descriptions nouvelles de l'Allemagne qui soient conformes à ce qu'elle est devenue. Celtis lui-même ne peut mener à bien ce projet, mais celui-ci inspire de nombreuses productions savantes et humanistes pendant tout le $\mathrm{XVI}^{\mathrm{e}}$ siècle, qui relèvent à la fois du genre de la chronique et de celui de la cosmographie: l'exemple le plus célèbre est celui de la grande Cosmographie (1544) de Sebastian Münster, dont le cœur est consacré à la description de l'Allemagne (Helmrath, Mulack et Walther, 2002 ; Burmeister, 1963, Besse, 2003). La Réforme protestante joue aussi un rôle dans l'introduction de l'enseignement géographique : elle conduit en effet à une réorganisation de l'enseignement dans les territoires passés au protestantisme, avant et après la paix d'Augsbourg (1555), qui impose le principe cujus regio ejus religio (la religion du prince détermine celle de ses sujets) et offre aux États du Saint Empire différents terrains d'affirmation de l'autonomie territoriale, en particulier dans le domaine de l'organisation ecclésiastique et scolaire (Heckel, 1983; Schindling et Ziegler, 1992). Cette réforme est largement influencée par la pensée de Philipp Melanchthon, le principal collaborateur de Luther mais également l'instigateur et le maître d'œuvre de la réorganisation des études à Wittenberg : il y gagne le surnom de 
Praeceptor Germaniae (le "précepteur de l'Allemagne ») (Hartfelder, 1889 et 1892 ; Scheible, 1997). Elle est notamment marquée, dans le domaine des facultés de philosophie, par l'importance qui est accordée aux savoirs mathématiques, parmi lesquels la géographie se trouve le plus souvent rangée. Ceux-ci constituent en effet, pour Melanchthon, un moyen de percevoir l'action de la Providence dans le monde, car Dieu organise la régularité des phénomènes naturels (notamment du cours des astres), mais donne aussi à l'homme la capacité rationnelle de les mesurer: pratiquer l'astronomie, la géométrie ou la géographie permet donc de renforcer la foi du chrétien (Kusukawa, 1995 ; Maurer, 1962 ; Bellucci, 1998 ; Methuen, 1998; Brosseder, 2004 ; Chassagnette, 2018). Le savoir géographique y est explicitement valorisé et encouragé, notamment parce qu'il permet d'illustrer, de comprendre et de mémoriser les récits historiques, en particulier ceux qui sont attachés à l'histoire sainte. Les universités et les écoles de l'Empire qui adoptent la Réforme ont souvent puisé au modèle melanchthonien, transmis notamment par la présence et l'activité des professeurs formés à Wittenberg ou proches de Melanchthon. Dans la plupart des cas, néanmoins, ce modèle est adapté aux exigences et aux traditions locales, qui peuvent reconnaître d'autres autorités dans la conception et l'organisation des savoirs.

3 Le présent texte propose d'évaluer l'importance de l'enseignement de la géographie à l'université de Marbourg, une institution de création moderne, dont la fondation même est étroitement corrélée à l'adoption de la Réforme protestante dans le landgraviat de Hesse. Il cherche à mesurer l'incidence de la réception de la réforme pédagogique de Wittenberg dans une institution protestante qui, comme d'autres, voit dans la ville et l'université de Luther une source essentielle, mais non exclusive, d'inspiration pour la constitution d'une éducation proprement réformée. Il s'agit enfin de mesurer l'introduction de l'enseignement de la géographie à l'aune de l'intérêt que gagne alors ce savoir dans les sphères lettrées de la Renaissance, mais également des enjeux confessionnels qui fondent et légitiment la mise en œuvre de ce domaine de connaissance.

\section{La fondation de l'université de Marbourg et la place de la géographie dans la première organisation de l'enseignement philosophique}

4 Marbourg est la première université fondée par un prince protestant: son acte de création date de 1527. En installant une université sur le territoire de Hesse, le landgrave Philipp manifeste l'étendue du pouvoir dont peut disposer un prince de l'Empire, indépendamment de l'empereur et avant même que les droits des territoires de l'Empire ne soient consolidés lors de la paix d'Augsbourg de 1555. L'université de Marbourg est à ce titre considérée par l'histoire allemande des institutions d'enseignement comme la première landesherrliche Universität (la première université territoriale sous autorité princière) (Gundlach, 1927; Schmitz, 1978). Pour Philipp de Hesse, la première fonction de l'université doit être de former des lettrés pour le service administratif et religieux du landgraviat dont la foi soit conforme à la doctrine luthérienne naissante. L'octroi d'un privilège impérial demeure nécessaire pour que l'institution obtienne la reconnaissance universelle de la collation des grades universitaires, qui, dans le contexte de la naissance de la Réforme, ne pouvait plus être obtenu du pouvoir pontifical. La confirmation impériale n'est intervenue qu'en 1541. 
Cette fondation précoce explique que l'influence de Wittenberg ne se soit pas exercée à Marbourg aussi directement que dans d'autres universités converties plus tardivement à la Réforme. De ce fait, l'enseignement des mathématiques et de la géographie y est resté longtemps moins valorisé qu'à Tübingen, Helmstedt ou Rostock.

L'enseignement de la géographie est, au XVI ${ }^{e}$ siècle, le plus souvent rattaché à celui des mathématiques, mais il arrive qu'il émerge également de l'enseignement de l'histoire, ou de lectures sur certains auteurs classiques. Il faut donc le plus souvent, pour espérer en avoir une idée assez précise, passer au crible l'intégralité des cours dédiés aux arts. Le premier règlement de l'enseignement dans la faculté de philosophie de Marbourg est établi le 31 août 1529, dans la Freiheitsbrief de Philipp de Hesse. Le programme est partagé en plusieurs chaires, dont une est attribuée à l'histoire et une autre aux mathématiques (alors que deux chaires de mathématiques existent à cette époque à la faculté des arts de Wittenberg). Il est prévu que le professeur d'histoire consacre ses cours aux auteurs classiques latins (Hildebrand, 1848). La liste établie n'est pas close et laisse à l'enseignant la liberté de choisir d'autres auteurs, mais aucune mention n'est faite de la géographie. Le programme proposé dans le cours de mathématiques n'est guère plus original : il prévoit l'enseignement des Éléments d'Euclide, du traité de la sphère de Sacrobosco, et de l'Astronomicon d'Hygin, manuel d'initiation à l'astronomie qui mélange connaissances physiques et légendes célestes. Cette relative simplicité de l'enseignement des mathématiques s'explique, entre autres, par la date à laquelle ont été élaborés ces statuts. En 1529, il n'est guère surprenant qu'aucun manuel de géographie ne soit mentionné, et moins encore que l'enseignement consacré à l'astronomie en reste à l'usage de manuels fondamentaux de la tradition médiévale. À cette date, l'enseignement de philosophie de Wittenberg commence à peine à être modifié sous l'influence de Melanchthon, et l'introduction du partage entre hautes et basses mathématiques, qui permet par la suite la diversification de l'enseignement, n'intervient qu'aux environs de 1525.

Un certain nombre d'ordonnances produites au XVI siècle à Marbourg ont pour but de réaménager l'administration et l'enseignement au sein de l'université et des différentes facultés. L'enseignement des mathématiques n'y est que très ponctuellement mentionné, le savoir géographique n'y est pas évoqué (Hildebrand, 1848 ; Cod. Hist. Lit. 120, Staats- und Universitätsbibliothek de Göttingen). L'enseignement des mathématiques à Marbourg est assuré par une chaire ordinaire unique, mais il semble que des chaires extraordinaires soient parfois venues s'y ajouter au cours du XVI ${ }^{\mathrm{e}}$ siècle.

Les professeurs de mathématiques à Marbourg (1527-1621)

\begin{tabular}{|l|l|}
\hline $1527-1528$ & Thomas Zeger \\
\hline $1531-1536$ & Buchardus Mithobius \\
\hline $1536-1538$ & Johannes Dryander \\
\hline $1538-1544$ & Nicolaus Wintrimius \\
\hline $1544-1553$ & Volcuinus Vigelius \\
\hline $1553-1554$ & Adam Lonicerus \\
\hline
\end{tabular}




\begin{tabular}{|c|c|}
\hline $1557-1591$ & Victorinus Schönfeld \\
\hline $1575-1578$ & Lazarus Schoner \\
\hline $\begin{array}{l}\text { Jusqu'en } \\
1598 ?\end{array}$ & $\begin{array}{l}\text { Rudolf Goclenius l'Ancien } \\
\text { [Par ailleurs professeur ordinaire de physique depuis } 1581 \text { et de logique depuis } \\
\text { 1589] }\end{array}$ \\
\hline $1592-1609$ & Johannes Hartmann \\
\hline $1609-1612$ & $\begin{array}{l}\text { Heinrich Hofmann } \\
\text { [+ cours extraordinaire de mathématiques pour Rudolf Goclenius le Jeune à partir } \\
\text { de } 1609 \\
\text { + enseignement par Rudolf Goclenius l'Ancien à partir de } 1609 \text { ?] }\end{array}$ \\
\hline $1612-1621$ & Rudolf Goclenius le Jeune \\
\hline
\end{tabular}

7 L'examen du tableau ci-dessus fait apparaitre un rythme rapide d'occupation de la chaire de mathématiques jusqu'au tournant $\mathrm{du} \mathrm{XVI}^{\mathrm{e}}$ siècle, période à laquelle Rudolf Goclenius l'Ancien commence à être chargé de cet enseignement, selon des modalités qui ne sont d'ailleurs pas très claires. Ces changements fréquents de titulaires de la chaire peuvent s'expliquer par le fait que sept d'entre eux ont obtenu un doctorat en médecine avant ou pendant leur enseignement de mathématiques. Il s'agit d'un type de parcours intellectuel et professionnel courant dans les universités européennes de la Renaissance, qui s'explique par l'attrait que pouvait exercer un titre académique qui ouvrait la voie à l'exercice d'un métier et à une chaire d'enseignement dans une université supérieure, souvent mieux rémunérée, et plus prestigieuse, que celle de mathématiques dans une faculté de philosophie (Chassagnette, 2018). Ce phénomène s'est trouvé considérablement atténué dans de nombreuses universités luthériennes, où l'enseignement mathématique revêt une importance nouvelle suite à la réforme pédagogique de Melanchthon à Wittenberg, et où sont recrutés, pour des durées assez longues, des professeurs dont la renommée est bien établie dans le domaine des mathématiques et de l'astronomie. Tübingen en est un bon exemple (Methuen, 1998; Hofmann, 1982). La situation diffère à Marbourg au XVI ${ }^{\mathrm{e}}$ siècle, où plusieurs professeurs de mathématiques sont ou deviennent médecins : c'est le cas pour Zeger, Mithobius, Blechler, Lonicerus, Schönfeld et Hartmann. Thomas Zeger est déjà docteur en médecine de l'université de Bâle lorsqu'il commence à enseigner les mathématiques à Marbourg. Dès 1528, il se consacre entièrement à la médecine : il est physicus (médecin) à Goslar, puis à Hambourg, et termine sa carrière comme professeur de médecine à Copenhague où il meurt en 1544 (Schmitz, 1978). Burchard Mithoff (Mithobius, 1501-1564) a étudié à Rostock et Erfurt avant d'être appelé à Marbourg. En 1535 il est docteur en médecine, et devient dès 1536 conseiller et médecin du landgrave Philipp à Cassel. En 1539, il est médecin du duc Erich de Brunswick à Münden puis de sa femme Elisabeth et de son fils. Il meurt à Münden (Janicke, 1885 ; Melchior, 1620). Johannes Dryander (1500-1560) a étudié à Erfurt, Bourges et Paris (où il a sans doute aussi enseigné les mathématiques et l'astronomie). Il devient médecin à Mayence en 1533 et entre au service de l'évêque Johannes von Metzenhausen à Coblence et Trèves. Il devient ensuite professeur à Marbourg, où il enseigne les mathématiques puis la 
médecine jusqu'à sa mort (Heerlinger, 1959). Nicolaus Blechler (Wintrimius) est maître ès arts de Marbourg avant 1530. En 1544 il devient médecin à Arndstadt, ce qui signifie qu'il a obtenu entre-temps un doctorat de médecine. Il meurt en 1566 à Erfurt (Schmitz, 1978). Volcuinus Weigel (Vigelius) est né vers 1516 à Wetter, et devient professeur de mathématiques à Marbourg en 1544, où il exerce aussi la fonction de doyen de la faculté de philosophie. Il est docteur en médecine en 1549, et devient médecin du landgrave Philipp à Cassel, où il meurt en 1579. Il a épousé la fille de l'imprimeur Egelnoff, chez lequel il publie plusieurs ouvrages, dont son introduction à l'arithmétique (Schmitz, 1978). Adam Lonitzer (Lonicerus, 1528-1586) obtient la maîtrise ès arts en 1545, devient professeur de mathématiques à Marbourg en 1553 et obtient la même année le doctorat en médecine. En 1554 il devient médecin de la ville de Francfort-sur-le-Main. Il a, comme Weigel, épousé une fille de l'imprimeur Egelnoff, chez lequel il publie plusieurs petits traités de médecine et surtout son œuvre majeure, consacrée à l'histoire naturelle (Naturalis historiae opus novum, 1555-1555) (Mägdefrau, 1987). Victorin Schönfeld (1525-1591) obtient le doctorat en médecine à l'université de Marbourg en 1556. En 1557 il devient professeur de mathématiques à la faculté de philosophie, en 1566 professeur de médecine. Il est l'auteur d'un traité médical sur la peste et d'un pronostic astrologique, publié à Wittenberg en 1562 (Winter, 1891).

Ces nombreuses réorientations (ou prolongements) de carrière expliquent donc que le cours de mathématiques de Marbourg ait fréquemment changé de titulaire. Le rythme rapide d'occupation de la chaire a pour corrélat probable le classicisme de l'enseignement mathématique dispensé : il s'agit d'une discipline que les professeurs enseignent en attendant mieux, et pas nécessairement en raison d'un intérêt ou d'un talent personnel dans ce domaine de savoir. De ce fait, l'enseignement est réduit aux traités fondamentaux et aux contenus de cours en usage à cette époque. L'étude des publications des occupants successifs de la chaire met ce fait en évidence. Adam Lonicerus (Lonitzer) est l'auteur d'une introduction à l'arithmétique à côté de nombreux traités de médecine, consacrés à la peste, aux sages-femmes ou à l'histoire naturelle (Lonitzer, 1551). Burchard Mithobius s'est intéressé à l'astronomie et a collaboré à quelques ouvrages de Johannes Dryander, mais n'a pas produit de manuel sur ce sujet à destination des étudiants. Dryander est peut-être, parmi les premiers professeurs de mathématiques de Marbourg, celui qui aurait pu être le plus intéressé à la géographie: médecin lui aussi, il a consacré beaucoup de temps aux recherches astronomiques, à côté de ses travaux sur l'anatomie. Il a également publié en 1537 une œuvre de Johannes Stöffler, les Cosmographicae aliquot descriptiones Ioannis Stofleri Iustingen Mathematici insignis : il s'agit d'un bref traité de géographie mathématique, qui décrit le découpage géométrique de la surface terrestre par les parallèles et méridiens, et expose des techniques de projection cartographique (Stöffler, 1537). Il est possible que Dryander ait utilisé ce traité dans son enseignement, mais rien ne le prouve non plus. La lettre dédicatoire de Dryander, adressée à Henricus Falcomontanus, rappelle de manière très conventionnelle l'utilité de la cosmographie, mais n'évoque pas de public étudiant ni la possibilité d'enseigner ce savoir à la jeunesse. 


\section{La diversification de l'enseignement mathématique et l'apparition de la géographie (dernier quart du XVIe siècle-1621)}

9 L'arrivée de Rudolph Goclenius l'Ancien à la chaire de mathématiques à la fin du XVI ${ }^{e}$ siècle marque le début d'une diversification de l'enseignement. L'incertitude qui entoure la date exacte des premiers cours de mathématiques de Goclenius est liée à la polyactivité de ce savant, qui a occupé de nombreuses chaires à la faculté de philosophie de Marbourg (Freundenthal, 1879). On sait qu'il a enseigné les mathématiques avant 1598 (sans doute à partir de 1590 environ), puis à nouveau à partir de 1609. On peut formuler quelques hypothèses sur le contenu de cet enseignement à partir des nombreuses disputes universitaires attribuées à Rudoph Goclenius et imprimées au XVI ${ }^{\mathrm{e}}$ siècle: ses cours ont traité de géométrie, de psychologie, de logique, d'optique, d'éthique, d'astronomie, de poétique, etc. (Goclenius, 1586, 1591 et 1592). La géographie n'y apparaît pas clairement. En revanche, Goclenius est l'auteur d'un manuel consacré à la cosmographie, les Cosmographiae seu sphaerae mundi descriptionis, qui traitent à la fois d'astronomie et de géographie (Goclenius, 1599). Son contenu est succinct et relève de la tradition cosmographique renaissante. Il a également composé un ouvrage dont le caractère généraliste semble indiquer qu'il s'agissait d'un manuel de cours, le Synopsis methodica. L'évocation de la géographie y est présente, mais également succincte (Goclenius, 1620). Il a enfin édité une encyclopédie et un lexique philosophiques, le Lexicon philosophicum, qui consacre quelques pages à la géographie et en donne une définition (Goclenius, 1613). Il ne s'agit pas d'une réflexion approfondie sur ce domaine de savoir, mais d'une brève synthèse sur la manière dont peut être caractérisée la géographie : elle n'y est pas définie comme savoir «mathématique ", "physique » ou « historique » (catégories sous lesquelles la plupart des traités de philosophie de l'époque classent le savoir géographique), mais plus simplement comme la «description de la Terre connue, qui est constituée de la chorographie et de la topographie ». Goclenius utilise les termes en usage au XVI e siècle et dans les premières décennies du XVII siècle pour définir la géographie, empruntant notamment le terme de "chorographie» (description régionale) à la tradition ptoléméenne, qui distingue la description de la Terre dans son ensemble et celle de ses parties. Le Lexicon philosophicum ne mentionne pas explicitement de public étudiant, mais la production d'encyclopédies, qui visent à recenser, organiser et transmettre l'ensemble des savoirs, est alors fréquente dans les institutions d'enseignement protestantes de l'Empire (Hotson, 2000). La publication de ces deux ouvrages de synthèse est postérieure à l'occupation de la chaire de mathématiques par Goclenius, mais ils peuvent traduire l'intégration de la géographie dans les cours de mathématiques dispensés jusqu'en 1598, sans qu'une place privilégiée ait été accordée à ce domaine de savoir.

10 Johannes Hartmann a occupé la chaire de mathématiques de 1592 à 1609, date à laquelle il obtient à Marbourg une chaire de chimie (chymitria), c'est-à-dire de pharmacopée dans une orientation paracelsienne de la médecine (Kerstein, 1966). Si les mathématiques semblent l'avoir beaucoup intéressé au moment de ses études de philosophie, toutes ses œuvres imprimées par la suite traitent de médecine et de chimie : il n'y a dans ses travaux aucune trace d'enseignement de la géographie. 
11 Le cas d'Heinrich Hofmann est un peu différent. Celui-ci n'a occupé que quelques années la chaire de mathématiques de Marbourg (de 1609 à 1612) et s'est, comme beaucoup de ses prédécesseurs, rapidement orienté vers la médecine (Vogel, 1972). Il a cependant poursuivi à Iéna son enseignement des mathématiques après 1613 , et surtout leur a consacré plusieurs travaux importants qui ont été imprimés, signe possible d'un travail pédagogique antérieur ou parallèle sur ces matières. Mathématicien reconnu au début du XVII e siècle pour sa traduction en allemand des Éléments d'Euclide et pour un ouvrage d'algèbre, Hofmann est aussi l'auteur d'un ouvrage sur l'octant, le De octantis Instrumenti (Hofmann, 1612). Ces publications montrent qu'il n'a pas cessé de s'intéresser aux mathématiques pendant ses études de médecine, et qu'il n'a pas non plus négligé leur versant appliqué. Le traité sur l'octant est dédié au landgrave Moritz de Hesse, qui portait beaucoup d'intérêt aux questions militaires. Hofmann présente l'octant (un huitième de cercle, proche parent du quadrant) comme un instrument utile dans des circonstances et des domaines très variés : c'est, rappelle-t-il dans la lettre dédicatoire, une caractéristique de la géométrie d'être un savoir nécessaire aux travaux les plus divers. Elle permet d'arpenter et de mesurer les villes, les îles, les régions, d'évaluer la hauteur des châteaux et de prévoir l'organisation des fortifications. Cette diversité d'usage est encore soulignée dans l'adresse au lecteur, où Hofmann semble viser un public élargi au-delà des horizons universitaires. Dans la lettre dédicatoire, la justification de l'usage de la géométrie rappelle les écrits de Melanchthon et de Caspar Peucer sur les mathématiques et leur relation à la Providence, et met en lumière la persistance, au sein des universités protestantes de l'Empire, de l'influence de la philosophie naturelle du Praeceptor Germaniae :

Est-il besoin d'en dire plus ? Parle, géométrie, non contente d'être terrestre et, pour ainsi dire sous nos pieds, tu nous fais aussi pénétrer le théâtre céleste, observer et expliquer les vestiges du très grand et très savant Architecte et Créateur du monde (Hofmann, 1612. Trad. A. Chassagnette).

Rudolph Goclenius le Jeune semble s'être un peu effacé devant la personnalité brillante de son père (Gundlach, 1927). Il a cependant enseigné assez longuement les mathématiques à Marbourg, de 1612 à sa mort en 1621. On ne s'étonne plus de le voir, lui aussi, entreprendre des études de médecine. De son père, avec lequel il a étroitement collaboré, il semble avoir hérité d'un goût pour la polymathie, avec un intérêt prononcé pour les doctrines proches de l'ésotérisme qui se développent parmi les professeurs d'université allemands au début du XVIIe siècle, en particulier dans les institutions calvinistes : ses publications ne touchent donc pas seulement à la médecine d'orientation paracelsienne et à la physiognomonie, mais aussi au magnétisme, à l'astrologie et à la théologie. En revanche, il n'a consacré aucune de ses œuvres au domaine des mathématiques.

\section{Jacob Müller et l'enseignement institutionnalisé de la géographie (1625-1637)}

L'enseignement que Jacob Müller a consacré à la géographie a pu être présenté comme la création d'une chaire de géographie à Marbourg. En réalité, Müller occupa à partir de 1625 une chaire de mathématiques (en plus d'une chaire de médecine) et c'est dans ce cadre qu'il s'intéressa à la géographie, et plus particulièrement à la géométrie 
appliquée et à l'arpentage (Folkert, 1997). L'apparition de cours très pratiques d'application de la géométrie (et en particulier de la trigonométrie dans la perspective de travaux de mesure des terrains) n'est pas un phénomène propre à l'université de Marbourg: on retrouve une évolution semblable dans de nombreuses universités protestantes du Saint Empire au début du XVII e siècle (Chassagnette, 2018). Les activités d'ingénieur, d'architecte et de conseiller militaire de Müller auprès du landgrave de Hesse-Darmstadt expliquent qu'il ait pu consacrer une part de son enseignement aux mathématiques appliquées, à une époque où, de surcroît, les débuts de la guerre de Trente ans incitaient les princes et les villes de l'Empire à rechercher des personnes compétentes en ces domaines. Par la diversité de ses talents et de ses activités (architecture, fortification, arpentage, dessin et gravure), le personnage de Müller peut être rapproché de celui de Wilhelm Dilich, au service du landgrave de Hesse-Cassel puis du prince-électeur de Saxe (Nieder, 2002 ; Baumgärtner, Stercken et Halle, 2011).

a crit plusieurs ouvrages, dont deux sont consacrés à la géométrie appliquée (Müller, 1619 et 1621). Le Compendium geometricum, rédigé en latin et paru en 1619, est sans doute destiné à un public d'étudiants, mais plus largement de lettrés. Il donne successivement des définitions de géométrie générale, des démonstrations et théorèmes, puis traite de géométrie plane et dans l'espace. La Praxis Geometria Universalis, parue en 1621, est rédigée en allemand et dédiée à un bourgeois de Giessen, le chevalier Johann-Melchior von Schwalbach. Le choix de la langue vernaculaire et du dédicataire indique que Müller vise ici un public large qui n'est pas limité à la sphère universitaire. L'ouvrage, quoique très clair, est assez complexe, notamment dans la démonstration des règles trigonométriques.

L'un et l'autre livre de Müller sont des traités généraux, conçus pour aider à l'application de la géométrie dans des circonstances pratiques diverses. Parmi les problèmes et exemples développés apparaissent l'arpentage, la mesure des superficies et des bâtiments, l'emplacement et l'orientation des fortifications, ou encore la construction de conduites d'eau, de pompes et de fontaines: toutes activités qui, rassemblées, requièrent les compétences d'un ingénieur polyvalent au service d'un prince ou d'une ville, et dont les fonctions s'institutionnalisent peu à peu au début du $\mathrm{XVII}{ }^{\mathrm{e}}$ siècle. Parmi ces compétences, celles du géographe, qui sait mesurer l'espace et en porter représentation sur une carte ou un plan, apparaissent comme indispensables. Les travaux de Müller mettent en évidence la conscience qu'ont les professeurs et les autorités universitaires de la polyvalence demandée aux mathématiciens, particulièrement recherchée dans le contexte militaire de la guerre de Trente ans. Il semble que l'enseignement s'y adapte, non seulement en élargissant et en diversifiant les cours de mathématiques, mais aussi en tentant de multiplier leurs domaines d'application.

16 Cette évolution de l'enseignement de la géographie vers les mathématiques appliquées s'éloigne nettement du contenu des cours de géographie proposés dans la plupart des universités luthériennes dans la seconde moitié du XVI ${ }^{e}$ siècle. Mais cette orientation n'a jamais été forte à Marbourg. Au début du XVII ${ }^{\mathrm{e}}$ siècle, en revanche, elle accompagne le tournant que prennent de nombreuses institutions d'enseignement protestantes allemandes dans l'enseignement des mathématiques, et à l'intérieur de ce dernier, de la géographie. À un savoir généraliste qui associe les fondements de la géographie mathématique, des travaux sur la mesure de la Terre, des calculs des coordonnées géographiques et d'autre part la lecture des descriptions du monde et de ses parties, 
s'est en partie substituée une géographie pratique, appliquée dans des contextes locaux à des travaux d'ingénierie, de gouvernement des territoires ou de fortification des villes, en réponse à la complexité et au développement croissants des outils d'administration des territoires et à un contexte militaire qui met à mal les territoires du Saint Empire. Dans cette perspective, les travaux des Anciens perdent de leur importance. La justification théologique qui présidait à l'enseignement de la géographie à Wittenberg dans la seconde moitié du XVIe siècle s'estompe également.

17 Reconstituer l'histoire de l'enseignement de la géographie dans une université allemande du XVI ${ }^{e}$ siècle relève d'un parcours souvent difficile qui, s'il ne veut se contenter des sources normatives (le plus souvent muettes sur ce sujet), doit chercher ailleurs les traces des contenus réels des cours dispensés : les ouvrages produits par les professeurs, les disputes philosophiques imprimées constituent à ce titre des ressources précieuses et permettent de retracer, parfois en pointillés, ce qu'a pu être l'enseignement dispensé. Marbourg n'échappe pas à la règle. Cette institution universitaire constitue néanmoins une exception dans le paysage des universités luthériennes de l'Empire. Fondée comme protestante au moment même où la doctrine luthérienne commence tout juste à se construire à Wittenberg, elle échappe en partie au modèle de la réorganisation des études qui se poursuit alors sous l'action de Philipp Melanchthon. L'université saxonne, sous l'autorité du Praeceptor Germaniae et de ses proches, accorde un intérêt tout particulier à la faculté des arts, à la philosophie naturelle et aux savoirs mathématiques (parmi lesquels la géographie). Cette réorganisation - elle-même mouvante dans les décennies suivantes et après la mort de Melanchthon en 1560 - inspire nombre d'universités des territoires luthériens de l'Empire. Il ne s'agit en aucun cas d'un modèle imposé, car la plupart de ces institutions dépendent de territoires différents, qui détiennent l'immédiateté impériale et une grande autonomie en matière de politique scolaire. Si influence il y a, elle est spirituelle, morale et intellectuelle, et s'exerce souvent par l'intermédiaire d'un ou de plusieurs enseignants, souvent proches de Melanchthon et formés en partie ou en totalité à Wittenberg. Dans certains cas, elle rejoint une tradition forte, et déjà installée, d'enseignement renforcé des savoirs mathématiques, de l'astronomie et de la géographie : c'est le cas à Tübingen, où Johannes Stöffler, qui a notamment formé Melanchthon avant son départ pour Wittenberg, a enseigné l'astronomie et la géographie de manière intensive de 1507 à 1531 : Sebastian Münster fut également un de ses élèves (Oestmann, 2013 ; Maurer, 1969). Ce transfert de modèle appuyé sur les relations interpersonnelles ou sur un intérêt individuel est précisément ce qui manque à Marbourg : aucun des professeurs qui occupent la chaire de mathématiques ne s'est formé à Wittenberg, et la majorité d'entre eux n'est pas restée longtemps dans cet enseignement, préférant le plus souvent le quitter pour exercer comme médecins auprès d'un prince, ou professeurs d'une faculté supérieure. Marbourg semble donc échouer à ce que d'autres universités protestantes allemandes sont parvenues à faire au cours du XVIe siècle : développer une politique qui accorde aux savoirs mathématiques, lesquels relèvent des facultés de philosophie et donc d'une logique propédeutique, une place suffisamment prestigieuse pour non seulement faire venir, mais encore conserver un savant qui y consacre son temps et sa renommée. L'université de Marbourg n'appuie pas la formation de ses futurs serviteurs sur la philosophie naturelle ni sur les savoirs mathématiques. En revanche, elle rejoint dès le début du XVII ${ }^{e}$ siècle une tendance qui caractérise la plupart des institutions protestantes de l'Empire (mais aussi d'autres établissements en Europe), en orientant une partie de son enseignement mathématique 
dans un sens plus pratique. Les outils géographiques trouvent alors à s'appliquer à des buts concrets, tels que l'arpentage, la construction ou la fortification. L'histoire des sciences a fréquemment expliqué ce changement par l'incidence, sur l'enseignement universitaire, de la nouvelle philosophie de la nature, adossée à l'expérience au détriment du respect des autorités anciennes, et à la mathématisation de la description du monde. La philosophie de Francis Bacon, qui marque profondément la pratique philosophique anglaise $\mathrm{du} \mathrm{XVII}^{\mathrm{e}}$ siècle, en est le représentant théorique le plus éminent. Mais cette philosophie est alors peu reçue en Allemagne, et n'est pas discutée à Marbourg. On ne peut guère plus invoquer les ambitions coloniales de l'Empire. Il est en revanche certain que les princes, dont l'autonomie s'affirme avec la confessionnalisation, ont plus volontiers recours aux compétences des savants pour connaître, exploiter et maîtriser leurs territoires. L'enseignement des facultés de philosophie s'adapte à ces nouveaux besoins, qui se renforcent après le début de la guerre de Trente ans en 1618. En ce sens, la confessionnalisation à l'université de Marbourg n'a pas joué, comme ailleurs, en inscrivant l'enseignement de la géographie (et plus largement des disciplines mathématiques) dans la construction de la culture luthérienne et le renforcement de la foi. En revanche, elle a joué en accroissant l'autonomie du landgraviat de Hesse et en mettant au jour les besoins croissants de cet État pour l'administration de son territoire.

\section{BIBLIOGRAPHIE}

Bellucci D., 1998, Sciences de la nature et Réformation. La physique au service de la théologie dans l'enseignement de Philippe Melanchthon, Rome, Vivere In, 717 p.

Besse J.-M., 2003, Les grandeurs de la Terre. Aspects du savoir géographique à la Renaissance, Lyon, ENS Éditions, $424 \mathrm{p}$.

Baumgärtner I., Stercken M. et Halle A. (dir.), 2011, Landtafeln hessischer Ämter zwischen Rhein und Weser, 1607-1625, Cassel, Kassel University Press, 240 p.

Burmeister K. H., 1963, Sebastian Münster - Versuch eines biographischen Gesamtbildes, Bâle, Stuttgart, Helbig \& Lichtenhahn, 211 p.

Broc N., 1986, La géographie de la Renaissance, 1460-1620, Paris, CTHS, 262 p.

Brosseder C., 2004, Im Bann der Sterne. Caspar Peucer, Philipp Melanchthon, und andere Wittenberger Astrologen, Berlin, Akademie Verlag, 292 p.

Chassagnette A., 2006, «La géométrie appliquée à la sphère terrestre. Le De dimensione terrae (1551) de Caspar Peucer », Histoire \& Mesure, 21, p. 7-28.

Chassagnette A., 2018, Savoir géographique et cartographie dans l'espace germanique protestant (1520-1620), Genève, Droz, 624 p.

Cormack L. B., 1997, Charting an Empire. Geography at the English Universities 1580-1620, Chicago, The University of Chicago Press, $298 \mathrm{p}$.

Dainville F. de, 1940, La géographie des humanistes, Paris, Beauschene, 562 p. 
Folkert M., 1997, « Müller », Neue Deutsche Biographie, 18, p. 312-313.

Freundenthal F., 1879, « Rudolph Goclenius », Allgemeine Deutsche Biographie, 9, p. 308-312.

Gallois L., 1890, Les géographes allemands de la Renaissance, Paris, E. Leroux, 266 p.

Gautier-Dalché P., 2009, La géographie de Ptolémée en Occident (IVe-XVIe siècle), Turnhout, Brepols, $443 \mathrm{p}$.

Goclenius l'Ancien R., 1586, Theses philosophicae ad disputandum in celeberrima augustissima academia marpugensi propositae, Herborn, Christoph Corvinus.

Goclenius l'Ancien R., 1591, Scholae seu disputationes physicae more academico more fere propositae, Marbourg, Paul Egelnoph.

Goclenius l'Ancien R., 1592, Theses meteorologicae cum nonnullis adjectis, Marbourg, Paul Egelnoph. Goclenius l'Ancien R., 1599, Cosmographiae seu sphaerae mundi descriptionis, hoc est Astronomiae et Geographiae Rudimenta, Marbourg, Kaspar Scheffer.

Goclenius l'Ancien R., 1613, Lexicon philosophicon, Francfort-sur-le-Main, veuve Matthias Becker.

Goclenius l'Ancien R., 1620, Synopsis Methodica Geometriae, Astronomiae, Astrologiae, Opticae et Geographiae, Francfort-sur-le-Main, Unckelius.

Gundlach F. (dir.), 1927, Catalogus professorum Academiae Marburgensis, 1527-1910, Marbourg, Elwert, $606 \mathrm{p}$.

Hammerstein N. et Buck A. (dir.), 1996, Handbuch der deutschen Bildungsgeschichte: 15-Jahrhundert. Von der Renaissance und der Reformation bis zum Ende der Glaubenkämpfe, Munich, Beck, 475 p.

Hartfelder K., 1889, Philipp Melanchthon als Praeceptor Germaniae, Berlin, Hofman, 46 p.

Hartfelder K., 1892, Melanchthonania Paedagogica. Eine Ergänzung zu den Werken Melanchthons im Corpus Reformatorum, Leipzig, Teubner, 315 p.

Heckel M., 1983, Deutschland im konfessionellen Zeitalter, Göttingen, Vandenhoeck \& Ruprecht, $298 \mathrm{p}$.

Herrlinger R., 1959, « Dryander », Neue Deutsche Biographie, 4, p. 142-143.

Helmrath J., Mulack U. et Walther G. (dir.), 2002, Diffusion des Humanismus. Studien zur nationalen Geschichtsschreibung europäischer Humanisten, Göttingen, Wallstein, 464 p.

Hildebrand B. (éd.), 1848, Urkundensammlung über die Verfassung und Verwaltung der Universität Marburg unter Philipp den Grossmütigen, Marbourg, Elwert, 110 p.

Hofmann H., 1612, De Octantis Instrumenti Mathematici Novi Geodaetis, Astronomis, Geographis, Iéna, héritiers Lippoldianus.

Hofmann N., 1982, Die Artistenfakultät an der Universität Tübingen, Tübingen, Mohr, 267 p.

Hotson H., 2000, Johann Heinrich Alsted 1588-1638: Between Renaissance, Reformation and Universal Reform, Oxford, Oxford University Press, 286 p.

Janicke K., 1885, « Mithoff », Allgemeine Deutsche Biographie, 22, p. 14-15.

Kerstein G., 1966, « Johannes Hartmann », Neue Deutsche Biographie, 7, 1966, p. 744.

Kusukawa S., 1995, The Transformation of Natural Philosophy: the Case of Philipp Melanchthon, Cambridge, Cambridge University Press, 268 p. 
Lestringant F., 1991, L'atelier du cosmographe ou l'image du monde à la Renaissance, Paris, Albin Michel, $270 \mathrm{p}$.

Lonitzer A., 1551, Arithmetices brevis et utilis introductio, Francfort-sur-le-Main, Christian Egelnoff.

Mägdefrau K., 1987, « Lonicerus », Neue Deutsche Biographie, 15, p. 147-148.

Maurer W., 1962, « Melanchthon und die Naturwissenschaften seiner Zeit », Archiv für Kulturgeschichte, 44, p. 199-226.

Maurer W., 1969, Der junge Melanchthon zwischen Humanismus und Reformation, vol 1 : « Der Humanist », Göttingen, Vandenhoeck \& Ruprecht, 864 p.

Melchior A., 1620, Vitae Germanorum medicorum, Heidelberg et Francfort-sur-le-Main, Johannes Georgius Geyder.

Methuen C., 1998, Kepler's Tübingen. Stimulus to a Theological Mathematics, Aldershot, Ashgate, $292 \mathrm{p}$.

Ms Cod. Hist. Lit. 120, Staats- und Universitätsbibliothek Göttingen.

Müller J., 1619, Compendium geometricum. Tres libro digestum, et tum theoricis, tum practicis propositionibus et demonstrationibus ad dimensionem Linearum, Figurarum, et Corporum necessariis, luculenter illustratum, Giessen, Caspar Chemlin.

Müller J., 1621, Geometrica Universalis, Das ist: Wie man alle Lineen und Figuren in corporibus Physicis aussmessen soll, Giessen, Caspar Chemlin.

Nieder H., 2002, Wilhelm Dilich (um 1571-1650). Zeichner, Schriftsteller und Kartograph im höfischem Dienst, Lemgo, Weserrenaissance-Museum Schloß Brake, 204 p.

Oestmann G., 2013, « Stöffler, Johannes », Neue Deutsche Biographie, 25, p. 387-388.

Paulsen F., 1885, Geschichte des gelehrten Unterrichts auf den deutschen Schulen und Universitäten vom Ausgang des Mittelalters bis zur Gegenwart, mit besonderer Rücksicht auf den klassischen Unterricht, Leipzig, Veit, $811 \mathrm{p}$.

Rüegg W. (dir.), 1996, Geschichte der Universität in Europa, vol. 2: « Von der Reformation zur Französischen Revolution (1500-1800) », Munich, Beck, 542 p.

Scheible H., 1997, Melanchthon, eine Biographie, Munich, Beck, 294 p.

Schindling A. et Ziegler W. (dir.), 1992, Die Territorien des Reichs im Zeitalter der Reformation und der Konfessionalisierung: Land und Konfession, 1600-1650, t. 4, Münster, Aschendorff, 288 p.

Schmitz R. (dir.), 1978, Die Naturwissenschaften an der Philipp-Universität Marburg, 1527-1977, Marbourg, Elwert, $540 \mathrm{p}$.

Shapin S., 1998, La révolution scientifique, Paris, Flammarion, 260 p.

Stöffler J., 1537, Cosmographicae aliquot descriptiones Ioannis Stofleri Iustingen Mathematici insignis, Marbourg, Eucharius Cervicorvus.

Strauss G., 1959, Sixteenth-Century Germany. Its Topography and Topographers, Madison, University of Wisconsin Press, 197 p.

Vogel K., 1972, « Heinrich Hofmann », Neue Deutsche Biographie, 9, p. 453-454.

Winter G., 1891, « Victorin Schönfeld », Allgemeine Deutsche Biographie, 32, p. 303. 


\section{RÉSUMÉS}

Cet article étudie le développement de l'enseignement de la géographie à l'université de Marbourg, dans le landgraviat de Hesse, entre 1527 et 1637. Première université protestante du Saint Empire, Marbourg a organisé son enseignement de philosophie en partie à distance de l'université de Wittenberg, depuis laquelle Philipp Melanchthon, principal collaborateur de Luther, a largement contribué à transformer les études des écoles et des universités protestantes de l'Empire. Cette particularité explique que l'enseignement des mathématiques et en particulier de la géographie à Marbourg se soit organisé sur un modèle différent de celui de la faculté de philosophie de Wittenberg. L'introduction d'un enseignement régulier de géographie y est plus tardive. Sa caractéristique principale est l'engagement, au tournant du $\mathrm{XVI}^{\mathrm{e}}$ siècle, vers une application pratique des savoirs géographiques, pour l'arpentage et l'ingénierie militaire notamment.

This paper examines the teaching of geography at the University of Marburg in the landgraviate of Hesse between 1527 and 1637. The faculty of arts of the University of Marburg - the first protestant university founded in the Holy Roman Empire - developped its own way of philosophical teaching and, for a significant part, remained free from the influence of Philipp Melanchthon. Melanchthon, the first collaborator of Martin Luther at Wittenberg, played a major role in organizing the new way of teaching in protestant schools and universities of the Holy Roman Empire. The relative independance of the University of Marburg explains why the teaching of mathematical disciplines, and especially of geography, did not follow the model of the philosophical faculty at Wittenberg. Geography was not regularly taught at Marburg until the end of the Sixteenth Century, and the orientation of the teaching was merely practical, especially applied to land surveying and military ingeneering.

\section{INDEX}

Keywords : teaching, geography, mathematics, university, Marburg, holy roman empire, Sixteenth Century

Mots-clés : enseignement, géographie, mathématiques, université, Marbourg, Saint-Empire, XVIe siècle

\section{AUTEUR}

\section{AXELLE CHASSAGNETTE}

MCF en histoire moderne, Université Lumière Lyon 2, chercheuse au LARHRA (UMR 5190)

LARHRA - MSH

14 avenue Berthelot

F-69363 Lyon cedex 07. Axelle.Chassagnette@univ-lyon2.fr 